\title{
STATISTICAL MECHANICS OF COMPRESSED SENSING
}

F. Krzakala et al., "Statistical physics based reconstruction in compressed sensing," Phys Rev. X 2, 021005 (2012).

F. Krzakala et al., "Probabilistic reconstruction in compressed sensing: algorithms, phase diagrams, and threshold achieving matrices," J. Stat. Mech.: Theory and Experiment, 8, 08009 (2012).

\section{Recommended and a Commentary by S.N. Coppersmith, University of Wisconsin}

Most signals are compressible because the amount of information in the signal is typically much smaller than than the number of bits in the signal (hence, the ubiquity of jpeg, gif, and zip files). The standard way to perform signal processing is to acquire the complete signal first, and then compress it. However, there are many situations where measurements are expensive, so that it would be highly advantageous to avoid acquiring the complete signal while still obtaining the relevant information. Compressed sensing enables one to do this [1].

For a compressible signal, there is a basis in which the relevant signal of $N$ bits is made up of $r$ ones and $N-r$ zeros, with $r \ll N$. (For instance, a message with blocks of many ones followed by blocks of many zeros is sparse in the basis in which the new "one" denotes a change in the original signal.) In compressed sensing, to determine both the basis in which the vector is sparse and the vector in the sparse basis, one takes $M$ measurements which are dot products of the signal (sent in any 
basis) with randomly chosen vectors, and then looks for a signal that minimizes both the error of the vector reconstruction and is maximally sparse. The field of compressed sensing took off when it was proven that successful reconstruction of a compressible signal is achieved tractably by minimizing the $L^{1}$ norm (sum of the absolute values of the entries of the vector) subject to the constraint that the dot products agree with the measurements, and, moreover, the reconstruction is robust to noise, for $M>r \log _{2} N[2,3]$.

The relevance of statistical physics to compressed sensing arises because the compressed sensing process is a minimization problem with quenched randomness which can be treated using the replica formalism used for spin glass problems $[4,5]$. The quenched disorder that is averaged over arises from the random vectors used for the dot products and/or from the choice of signal. In the limit $M, N \rightarrow \infty$, there is a phase transition as a function of $\alpha=M / N$; there is a critical value $\alpha_{c}$ above which the reconstruction succeeds in reconstructing the original vector with high probability; for $\alpha<\alpha_{c}$ the reconstruction fails with high probability.

The statistical mechanical approach does not just provide another way of interpreting known results; it has enabled compressed sensing to be improved. The papers by Krzakala et al. [6, 7] show that (1) message-passing algorithms similar to those used to solve satisfiability problems can be used to do the optimization process more simply and efficiently than previous proposals, and (2) the reason why $r \log _{2} N$ samples are needed (instead of the $r$ samples that are strictly necessary to encode the amount of information present in the signal) is that the optimization algorithms get "stuck" in a metastable state, just as a statistical mechanical system near a first-order transition 
can get "stuck" in a phase with higher free energy until there is nucleation of the lower free energy phase. Krzakala et al. present an algorithm that "nucleates" successful reconstruction and achieves the asymptotically optimal result that the number of measurements needed is $r$ instead of $r \log _{2} N$. Thus, statistical mechanics techniques not only yield new insight into compressed sensing, but they also provide a means for improving the performance of the technique.

\section{REFERENCES}

[1] Dana Mackenzie, "Compressed sensing makes every pixel count", What's Happening in the Math. Sciences, AMS, 114-127 (2009).

[2] Emmanuel Candès, Justin Romberg, and Terence Tao, "Robust uncertainty principles: Exact signal reconstruction from highly incomplete frequency information," IEEE Trans. on Information Theory, 52, 489-509 (2006)

[3] David Donoho, "Compressed sensing," IEEE Trans. on Information Theory, 52, 1289-1306 (2006).

[4] Y. Kabashima, T. Wadayama, and T. Tanaka, "A typical reconstruction limit for compressed sensing based on L p -norm minimization," J. Stat. Mech.: Theory and Experiment 2009, L09003 (2009).

[5] S. Ganguli and H. Sompolinsky, "Statistical mechanics of compressed sensing," Phys. Rev. Lett. 104, 188701 (2010)

[6] F. Krzakala et al., "Statistical physics based reconstruction in compressed sensing," Phys Rev. X 2, 021005 (2012).

[7] F. Krzakala et al., "Probabilistic reconstruction in compressed sensing: algorithms, phase diagrams, and threshold achieving matrices," J. Stat. Mech.: Theory and Experiment, 8, 08009 (2012). 